\title{
Asymmetry Driven Phase Transformations
}

\author{
J. DAMCZYK ${ }^{a *}$, K. Ostasiewicz ${ }^{a}$, Ł. RAdosiński ${ }^{b}$ And A. RAdosz $^{a}$ \\ ${ }^{a}$ Institute of Physics, ${ }^{b}$ Institute of Physical and Theoretical Chemistry \\ Wrocław University of Technology, Wybrzeże Wyspiańskiego 27, 50-370 Wrocław, Poland
}

(Received November 13, 2009; in final form March 25, 2010)

\begin{abstract}
Motivated by the properties of one-dimensional lattice systems with asymmetric on-site potential, one can formulate a hypothesis of an asymmetry driven phase transformation. Characteristic feature of one-dimensional systems exhibiting asymmetry driven phase transformation is a sequence of the two phase conversions. In particular class of such systems with a triple-well potential, phase conversions of one-dimensional systems would evolve into a sequence of two phase transitions in three-dimensional models. We propose here a model of three-dimensional system exhibiting a sequence of two first order asymmetry driven phase transitions.
\end{abstract}

PACS numbers: 05.70.-a, 64.60.-i, 63.70.+h

\section{Motivation}

In the thermodynamic regime, one-dimensional (1D) lattice systems with degenerated vacuum, reveal the coexistence of localized and extended excitations, kinks and phonons, respectively, that is accompanied by characteristic hump developed in a specific heat $[1,2]$. Three-dimensional (3D) version of such a system exhibits phase transition of second order. In fact, it is one of the simplest manifestations of spontaneously broken (discrete) symmetry.

It has been recently observed $[3,4]$ that one-dimensional, multistable systems, with non-degenerated vacuum would exhibit interesting behavior. Specific heat of systems with local asymmetric, double-well or triple-well potential, may reveal quite rich, two-peak structure, possibly corresponding to two phase conversions. One can ask whether such a property of one-dimensional systems, would indicate a sequence of the phase transitions in corresponding three-dimensional systems.

The aim of this paper is to give a comprehensive analysis of unconventional behaviour of specific heat of a class of one-dimensional lattice systems with triple-well potential. It is argued that large, shape-type asymmetry of these systems is manifested by "energy level crossing", associated with two-peak structure of specific heat. In this case it reflects a sequence of two phase conversions. These phase conversions would turn into phase transitions in three-dimensional version of the model. We propose here a model of a three-dimensional lattice system exhibiting a sequence of two phase transitions - they may be referred to as asymmetry driven phase transitions. The

\footnotetext{
* corresponding author; e-mail: jacek.damczyk@pwr.wroc.pl
}

existence of such a sequence of phase transitions is far from being common phenomena among lattice systems with on-site multistability. Namely, in a widely used " $\phi$ " model asymmetry driven phase transitions cannot be observed as this potential does not reveal shape asymmetry property.

\section{Sequence of phase conversions - 1D systems}

Let us consider one-dimensional, one-component system of harmonically coupled atoms with a local multistability represented by a triple-well potential. The system is described by the Hamiltonian of the form [1-3]:

$$
H=\frac{m \omega_{0}}{a^{2}}\left[\sum_{l}\left(\frac{1}{2} \dot{x}_{l}^{2}+V\left(x_{l}\right)+\frac{1}{2} k\left(x_{l}-x_{l+1}\right)^{2}\right)\right],
$$

where $a$ denotes the characteristic inverse distance, $\omega_{0}$ characteristic frequency, and $m$ is the characteristic mass i.e. mass of single atom in the chain; $x_{l}$ is dimensionless coordinate at $l$-th site of the chain $(N$-sites with a periodic boundary conditions), $\dot{x}_{l}$ denotes (dimensionless) time derivative, and $k$ is dimensionless coupling constant. Thermodynamics of system (1) may be found by means of the transfer integral method, in continuum, classical limit [2-4]. Namely, the thermodynamic properties are related to the pseudo-Schrödinger eigenvalue problem

$$
\begin{aligned}
& \left(-\frac{1}{2 m^{*}} \frac{\mathrm{d}^{2}}{\mathrm{~d} x^{2}}+V(x)+V_{0}(T)\right) \Psi_{i}(x)=E_{i} \Psi_{i}(x), \\
& m^{*}=k T^{-2}, \quad V_{0}(T)=-\frac{T}{2} \ln \left(\frac{2 \pi T}{k}\right),
\end{aligned}
$$

where $T=k_{\mathrm{B}} \theta / \frac{m \omega_{0}}{a^{2}}$ is dimensionless temperature $\left(k_{\mathrm{B}}\right.$ - the Boltzmann constant, $\theta$ - temperature). Config- 
uration part of free energy is expressed via ground state "energy" of the eigenvalue problem (2)

$$
F_{\text {con }} \approx E_{0}(T), \text { for } k \gg 1 \text {. }
$$

One can choose potential $V(x)$ of particular shape: central well $(\mathrm{CW})$ is narrower and deeper than symmetric side wells (DW - double well) (see Fig. 1).

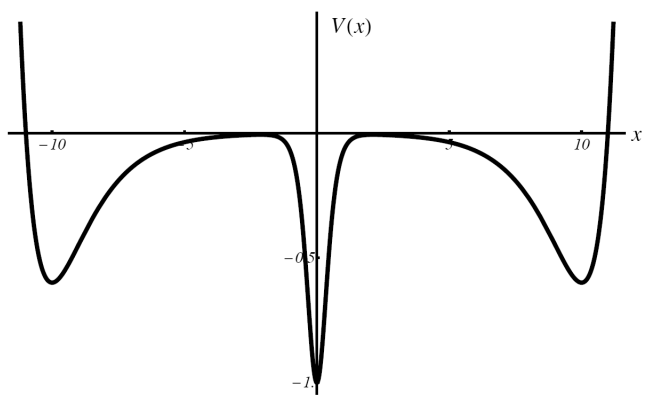

Fig. 1. On-site potential $V(x)$ (Eq. (4)) with parameters $V_{\mathrm{DW}}: V_{1}=V_{2}=0.6, \alpha=\beta=0.7, p=10.0$, and $V_{\mathrm{CW}}: U=1.0, \kappa=2.0$.

We take here $V(x)$ as composed of a double-Morse potential (DW), $V_{\mathrm{DW}}$ and Pöshl-Teller potential, $V_{\mathrm{CW}}$ :

$$
\begin{aligned}
& V(x)=V_{\mathrm{DW}}(x)+V_{\mathrm{CW}}(x), \\
& V_{\mathrm{DW}}(x)=V_{1}[\exp (-2 \alpha(x+p))-2 \exp (-\alpha(x+p))] \\
& \quad+V_{2}[\exp (-2 \beta(x-p))-2 \exp (-\beta(x-p))], \\
& V_{\mathrm{CW}}(x)=\frac{-U}{\cosh ^{2}(\kappa x)} .
\end{aligned}
$$

Specific heat of the system (1), (4)-(6), obtained from Eq. (3) is shown in Fig. 2. Asymmetry of the local po-

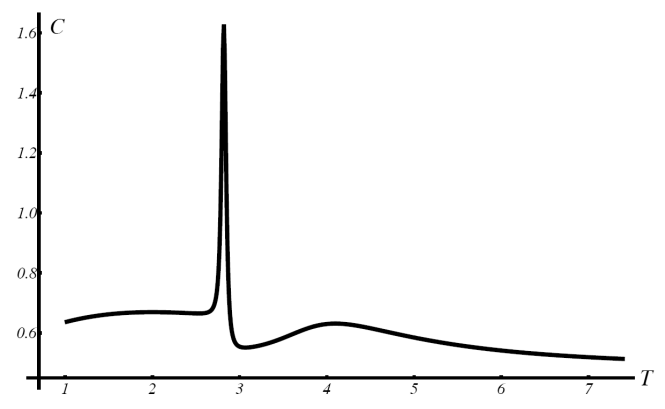

Fig. 2. Specific-heat $C$ versus temperature $T$ for $k=$ 10.0, and with on-site potential parameters as in Fig. 1.

tential leads to a rich structure of this function: apart of smooth hump, in higher temperature range, sharp and intensive peak, in low temperature range, manifesting dramatic change in the system, is developed in this case of large, shape-type asymmetry. Interpretation of this type of specific heat temperature dependence, given within semiclassical approach, real trajectories in complex time (RTCT) [3, 4] is the following. Free energy of a more stable phase, corresponding to lower energy within CW, is increasing faster than the free energy of the less stable phase, corresponding to higher energy within DWs. These two intersect at some temperature $T_{0}$ and at higher temperatures, $T>T_{0}$, DW becomes dominant phase as corresponding free energy is lower than the free energy of CW phase. Particles, occupying at low temperature range $\mathrm{CW}$, perform radical jump to a shallower, side well(s) of DW structure; this jump corresponds to a sharp peak of specific heat. Final phase conversion, related to the hump of a specific heat is associated with transition to the "disordered" phase: particles move from the side wells to the central well, where they are oscillating about central, $x=0$, position. This description is confirmed by means of numerical tools. Numerical analysis of the free energy (3) provides two characteristic features in this case. First, the temperature of former, low-temperature phase conversion, $T_{0} \approx 2.85$ (see Fig. 2 ), corresponds to the (avoided) crossing of the free energies of CW and DW phases ("energy level crossing" [3, 4], see Fig. 3).

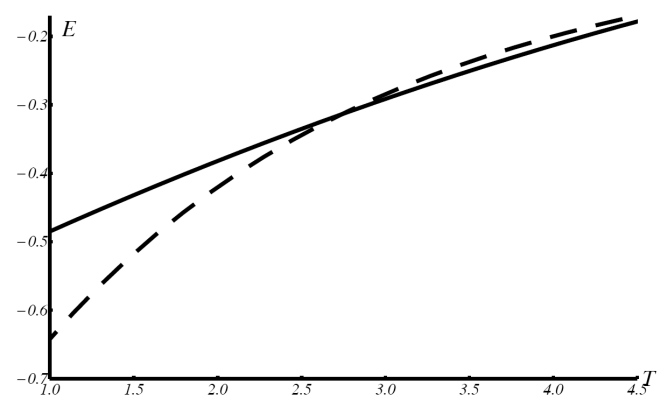

Fig. 3. The ground "energy" levels crossing for on-site potential: $V_{\mathrm{DW}}$ - solid line, $V_{\mathrm{CW}}$ - dashed line for $k=10.0$, and with parameters as in Fig. 1 .



Fig. 4. Specific heat $C$ versus temperature $T$ with distinct contributions. ( $a$ ) dashed line $V_{\mathrm{CW}},(b)$ dash-dot-dotted line $V_{\mathrm{DW}},(c)$ solid line full $V(x)$ for $k=10.0$, and on-site potentials parameters: $V_{\mathrm{DW}}: V_{1}=V_{2}=$ $0.75, \alpha=\beta=0.7, p=10.0, V_{\mathrm{CW}}: U=1.0, \kappa=2.0$.

Second, investigating the specific heat structure (Fig. 4) one can notice that it is composed of three distinct contributions. In the low- and high-temperature regimes (a), it reveals the shape characteristic of $\mathrm{CW}$; at the intermediate temperatures (b), a hump corresponding to a double-well structure, though shifted is visible; as added 


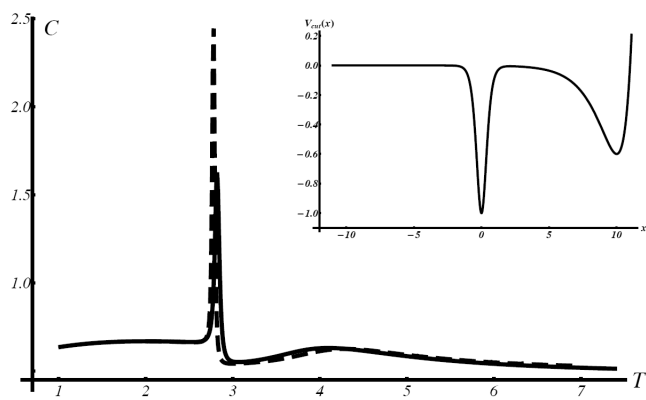

Fig. 5. Specific heat $C$ versus temperature $T$ for: full $V(x)$ (solid line), and one with one removed side-well $V_{\text {cut }}(x)$ (dashed) for $k=10.0$, and on-site potentials parameters: $V(x)$ as in Fig. 1 , and $V_{\text {cut }}(x)$ as given by $V_{\mathrm{DW}}: V_{1}=0, V_{2}=0.6, \alpha=0, \beta=0.7, p=10.0$, and $V_{\mathrm{CW}}: U=1.0, \kappa=2.0$. Inset: plot of the $V_{\text {cut }}(x)$ on-site potential with above parameters.

together (c), these two parts fit to the specific heat of the system. Sharp and narrow peak is missing in (a) and (b) as it arises when the central well and side wells, at least one of them (see below), are present together. Key element of the two-peak structure of the specific heat becomes evident when one removes one of the side wells. In this case, Fig. 5, one finds only small quantitative correction in the specific heat. This indicates that the asymmetry between narrow, deeper well and wider, shallower one, is the key feature of the on-site potential.

\section{Sequence of phase transitions - 3D systems}

Phase transformations, described above as a sequence of two consecutive transitions between "central-well" phase, stable at low- and high-temperature ranges, and "side-wells" phase, stable at the finite temperature range, manifested in specific heat of $1 \mathrm{D}$ systems would evolve into phase transitions in 3D systems. Let us consider 3D lattice system with on-site triple-well asymmetric potential of shape asymmetry. To make the problem tractable (by means of some self-consistent approximation), we propose here on-site potential as composed of " $\phi$ " as the DW, and inverted Gaussian as well the CW (see also [5]):

$$
\begin{aligned}
& H=U_{0}\left[\sum_{\boldsymbol{l}}\left(\frac{1}{2} \dot{u}_{\boldsymbol{l}}^{2}+V\left(u_{\boldsymbol{l}}\right)\right)\right. \\
& \left.+\frac{1}{4} \sum_{\boldsymbol{l}, \boldsymbol{l}^{\prime}} \varphi_{\boldsymbol{l} \boldsymbol{l}^{\prime}}\left(u_{\boldsymbol{l}}-u_{l^{\prime}}\right)^{2}\right], \\
& V(u)=V_{\mathrm{DW}}(u)+V_{\mathrm{CW}}(u), \\
& V_{\mathrm{DW}}(u)=-\frac{1}{2} u^{2}+\frac{1}{4} u^{4}, \\
& V_{\mathrm{CW}}(u)=-\gamma \exp \left(\frac{-u^{2}}{2 \delta^{2}}\right), \\
& f_{0}=\sum_{\boldsymbol{l}, \boldsymbol{l}^{\prime}} \varphi_{\boldsymbol{l} \boldsymbol{l}^{\prime}} .
\end{aligned}
$$

Energy barrier height of " $\phi$ " " potential, $U_{0}$ is taken as a common energy parameter, so dimensionless notation for other parameters and variables of model (7) is applied. Namely $\varphi_{\boldsymbol{l} \boldsymbol{l}^{\prime}}$ denotes harmonic coupling of atoms occupying lattice sites $\boldsymbol{l}$ and $\boldsymbol{l}^{\prime}$, where $\boldsymbol{l}=\left(l_{x}, l_{y}, l_{z}\right)$. Central well depth and wideness parameters are denoted as $\gamma$ and $\delta$, respectively. In $1 \mathrm{D}$ version of model $(7 \mathrm{a})$, the energy levels intersection is expected to occur in the following range of parameters (see [6]):

$$
\gamma>\frac{1}{4}, \quad \delta<0.3
$$

As argued above, level intersection corresponds to the narrow peak appearance, followed by the moderate peak in specific heat. Therefore the system reveals shape asymmetry and one can expect three-dimensional version (7a) would exhibit a sequence of the two phase transitions. System (7a), treated in terms of mean field approximation satisfies these expectations. Namely, for the triple-well potential parameters, belonging to the range (8) the system (7a) shows an unconventional behavior exhibiting two phase transitions. The temperature dependence of the order parameter in this case is shown in Fig. 6. As one can see in this figure, the system uncovers

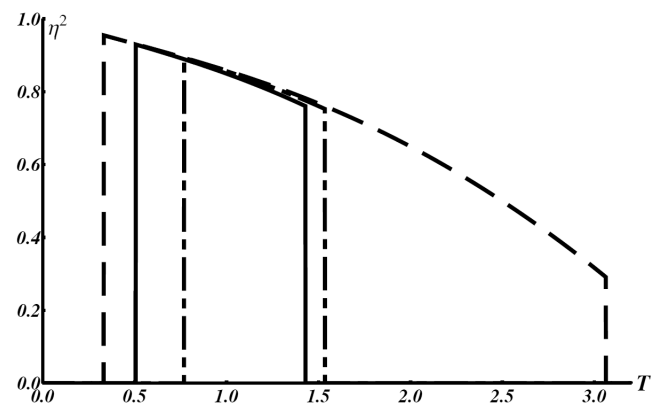

Fig. 6. Square of order parameter $\eta^{2}$ vs. temperature $T$ for $f_{0}=20$, and on-site potential $V(x)$ (Eq. (7b)) with parameters: $\gamma=0.28, \delta=0.185$ - solid line; $\gamma=0.30, \delta=0.14$ - dashed line; $\gamma=0.32, \delta=0.15-$ dash-dotted line.

the existence of an ordered phase in the finite temperature range. Both phase transitions are of the first order (though in this approximation the high-temperature phase transition might be of the second order). The detailed discussion of the asymmetry driven phase transition in model (7a) will be given in a more extended paper [6].

\section{Conclusions}

Shape asymmetry of one-dimensional lattice systems, associated with the "energy level crossing" and in consequence with a sequence of two phase conversions, may, in particular cases, lead to a sequence of phase transitions for the three-dimensional versions of the models. Detailed analysis of the specific heat function of $1 \mathrm{D}$ systems, has led to the formulation of the three-dimensional lattice model with triple-well, on-site potential uncovering shape asymmetry. Order parameter, of such a system, 
derived within mean field approximation, takes nonzero value at finite range of temperatures, indicating two first order phase transitions. Is a condition of shape asymmetry, satisfactory one for the sequence of asymmetry induced phase transitions, becomes as yet, an unanswered question. This problem will be discussed in our following paper, where the properties of the potential $(7 \mathrm{~b}),(7 \mathrm{c})$, (7d) and the thermodynamic properties of the system (7a) will be investigated. Here it should be pointed out that though variety of lattice systems with multistable local potential have been studied (see e.g. [7, 8]) this kind of behavior, asymmetry driven phase transitions, has not been reported. The reason is following. Shape asymmetry, that appears to be a necessary condition is neither universal nor common feature of multistable potentials. The well-known and widely studied example of a triple well-potential is the so-called " $\phi$ " model

$$
V(x)=\frac{A}{2} x^{2}-\frac{B}{4} x^{4}+\frac{D}{6} x^{6} .
$$

Interplay among parameters $A, B$ and $D$ would re-shape $V(x)(9)$. It is found, however, that a shape asymmetry, leading to central well and side wells energy levels crossing could not be yielded here. " $\phi$ " potential does not reveal shape asymmetry and that is why such a system has not been found to reveal a sequence of two first order phase transitions.

\section{References}

[1] A. Khare, S. Habib, A. Saxena, Phys. Rev. Lett. 79, 3797 (1997).

[2] J. Currie, S. Trullinger, A. Bishop, J. Krumhansl, Phys. Rev. B 15, 5567 (1977).

[3] A. Radosz, K. Ostasiewicz, P. Machnikowski, P. Magnuszewski, Phys. Rev. E 64, 062103 (2001).

[4] A. Radosz, K. Ostasiewicz, P. Magnuszewski, Ł. Radosiński, J. Damczyk, A. Mituś, G. Pawlik, F. Kusmartsev, J. Samson, Phys. Rev. E 73, 026127 (2006).

[5] N.M. Plakida, Fiz. Nizk. Temp. 7, 644 (1981) (in Russian).

[6] K. Ostasiewicz, J. Damczyk, Ł. Radosiński, A. Radosz, to be published.

[7] A. Khare, A. Saxena, J. Math. Phys. 49, 063301 (2008).

[8] K. Ji, K. Namikawa, H. Zheng, K. Nasu, Phys. Rev. B 79, 144304 (2009). 